Supporting Information

\title{
Simultaneous detection of ruthenium and osmium by photochemical vapor generation-inductively coupled plasma mass spectrometry
}

Qing Yang ${ }^{1,3 \#}$, Hanjiao Chen ${ }^{2 \#}$, Jing $\mathrm{Hu}^{2, *}$, Ke Huang ${ }^{3}$, Xiandeng Hou ${ }^{1,2, *}$

${ }^{1}$ Key Laboratory of Green Chemistry \& Technology of MOE, College of Chemistry, Sichuan University, Chengdu, Sichuan 610064, China

${ }^{2}$ Analytical \& Testing Center, Sichuan University, Chengdu, Sichuan 610064, China.

${ }^{3}$ College of Chemistry and Material Science, Sichuan Normal University, Chengdu, Sichuan 610068, China

Qing Yang and Hanjiao Chen contributed equally to this work.

Corresponding authors’E-mails: houxd@scu.edu.cn; hujing2019@scu.edu.cn 


\section{Table of Contents}

Figure S1. Feasibility study

Table S1. Instrumental parameters of ICP-MS

Table S2. Effect of common cations and anions on the simultaneous PVG of Ru and Os.

Table S3. Analytical results of water samples.

Table S4. Comparison of the figures of merit for Os detection by PVGICPMS/ICPOES. 

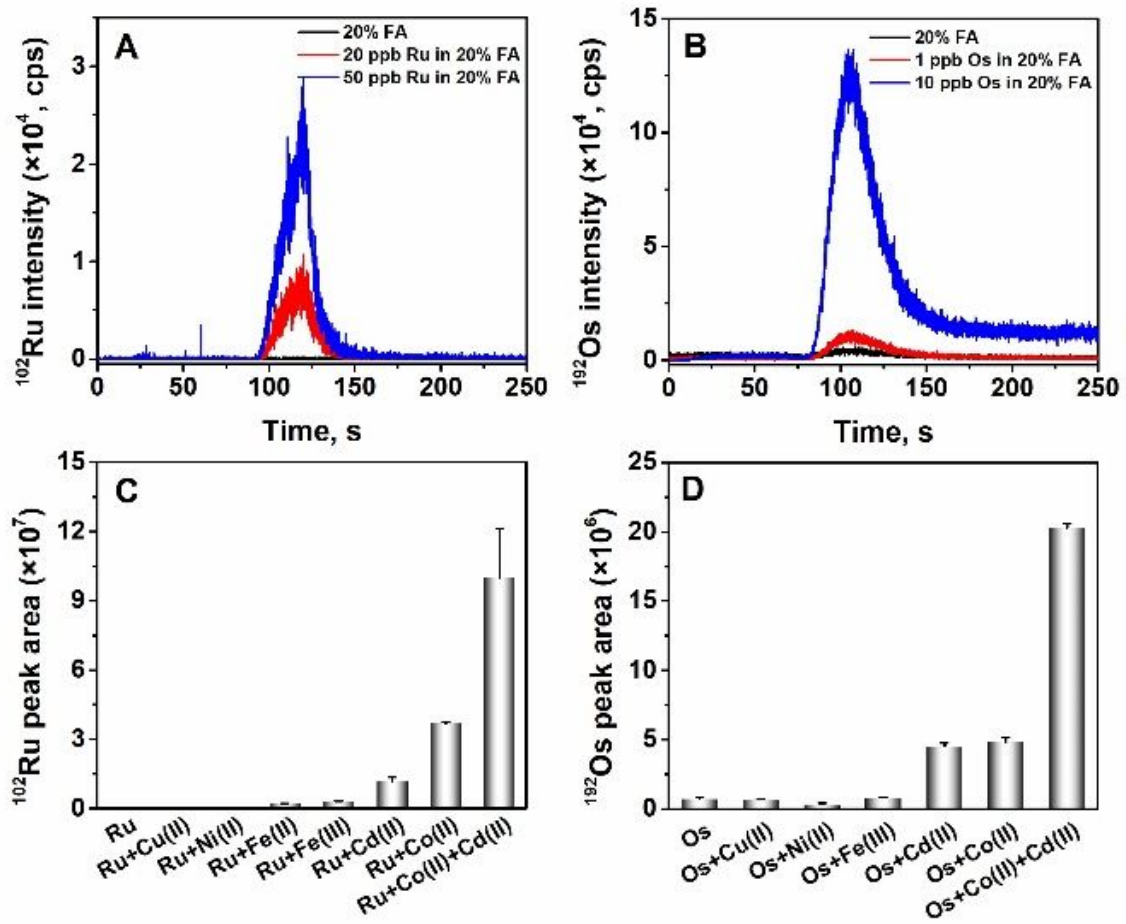

Figure S1. Typical PVG-ICP-MS signals from: (A) $20 \mu \mathrm{g} \mathrm{L} \mathrm{L}^{-1}$ and $50 \mu \mathrm{g} \mathrm{L} \mathrm{L}^{-1} \mathrm{Ru}$; and (B) $1 \mu \mathrm{g} \mathrm{L}^{-1}$ and $10 \mu \mathrm{g} \mathrm{L} \mathrm{L}^{-1}$ Os, generated from $20 \%$ formic acid. Effect of metal ions on the PVG efficiency of: (C) $10 \mu \mathrm{g} \mathrm{L}-1$ of Ru and (D) $2 \mu \mathrm{g} \mathrm{L} \mathrm{L}^{-1}$ of Os. Spiked metal ions levels were $20 \mathrm{mg} \mathrm{L}^{-1}$ for $\mathrm{Ni}(\mathrm{II}), \mathrm{Fe}(\mathrm{II}), \mathrm{Fe}(\mathrm{III}), \mathrm{Cd}(\mathrm{II}), \mathrm{Co}(\mathrm{II})$ and $2 \mathrm{mg} \mathrm{L}^{-1}$ for $\mathrm{Cu}(\mathrm{II})$. The UV light exposure time and argon carrier gas flow rate were kept constant at $60 \mathrm{~s}$ and $100 \mathrm{~mL} \mathrm{~min}^{-1}$, respectively. Uncertainties expressed as standard deviation $(\mathrm{n}=3)$.

Table S1. Instrumental parameters of ICP-MS

\begin{tabular}{ll}
\hline Conditions & ICP-MS (Thermo iCAP Q) \\
\hline Measurement mode & KED (collision helium gas on) \\
Isotopes measured (dwell time) & ${ }^{101} \mathrm{Ru}(10 \mathrm{~ms}),{ }^{102} \mathrm{Ru}(10 \mathrm{~ms})$, \\
& ${ }^{189} \mathrm{Os}(10 \mathrm{~ms}),{ }^{192} \mathrm{Os}(10 \mathrm{~ms})$ \\
Cool gas & $\mathrm{Ar}, 14.0 \mathrm{~L} / \mathrm{min}$ \\
Auxiliary gas & $\mathrm{Ar}, 0.8 \mathrm{~L} / \mathrm{min}$ \\
Nebulizer gas & Ar, $1.07 \mathrm{~L} / \mathrm{min}$ \\
Analysis mode & tQuant \\
RF forward power & $1550 \mathrm{~W}$ \\
\hline
\end{tabular}


Table S2. Effect of common cations and anions on the PVG of Ru and Os.

\begin{tabular}{cccc}
\hline \multirow{2}{*}{$\begin{array}{c}\text { Interfering } \\
\text { ions }\end{array}$} & $\begin{array}{c}\text { Amount added } \\
\mu \mathrm{g} / \mathrm{L}(\mathrm{mM})\end{array}$ & \multicolumn{2}{c}{ Recovery $(\% ; \mathrm{n}=3)$} \\
\cline { 3 - 4 } $\mathrm{Na}$ & 2000 & $90.8 \pm 1.3$ & Os \\
$\mathrm{K}$ & 2000 & $90.9 \pm 1.3$ & $95.0 \pm 2.6$ \\
$\mathrm{Ca}$ & 2000 & $91.4 \pm 0.2$ & $105.8 \pm 1.2$ \\
$\mathrm{Mg}$ & 2000 & $101.2 \pm 0.2$ & $108.5 \pm 1.3$ \\
$\mathrm{Zn}$ & 2000 & $93.3 \pm 2.0$ & $102.0 \pm 1.9$ \\
$\mathrm{Ni}$ & 2000 & $103.7 \pm 4.1$ & $97.7 \pm 1.7$ \\
$\mathrm{Mn}$ & 2000 & $95.7 \pm 1.5$ & $100.4 \pm 3.0$ \\
$\mathrm{Fe}$ & 2000 & $96.9 \pm 1.7$ & $95.2 \pm 0.7$ \\
$\mathrm{Cu}$ & 1000, & $73.0 \pm 3.0$, & $91.7 \pm 1.2$ \\
$\mathrm{Hg}$ & 500,200 & $107.4 \pm 1.8,107.4 \pm 1.8$ & $75.3 \pm 0.5,92.2 \pm 1.8$ \\
$\mathrm{As}$ & 500,200 & $83.5 \pm 4.0,88.0 \pm 2.9$ & $90.7 \pm 5.2,89.7 \pm 1.8$ \\
$\mathrm{SO}_{4}{ }^{2-}$ & 500,200 & $87.8 \pm 1.2,98.5 \pm 0.9$ & $75.4 \pm 2.5,88.9 \pm 1.1$ \\
$\mathrm{PO}_{4}{ }^{3-}$ & $(0.5,0.1)$ & $96.8 \pm 2.6,100.2 \pm 32$ & $88.7 \pm 2.2,91.6 \pm 2.4$ \\
$\mathrm{Cl}^{-}$ & $(0.5,0.1)$ & $96.1 \pm 1.7,95.4 \pm 3.4$ & $89.4 \pm 1.7,88.4 \pm 2.4$ \\
& $(0.5,0.1)$ & $97.8 \pm 2.8,98.3 \pm 1.3$ & $97.2 \pm 0.8,100.0 \pm 0.5$ \\
$\mathrm{NO}_{3}{ }^{-}$ & $(0.5,0.1)$ & $82.3 \pm 2.1,90.1 \pm 1.7$ & $65.9 \pm 1.6,84.1 \pm 3.0$ \\
\hline
\end{tabular}

Table S3. Analytical results of Ru and Os in real water samples (ng/mL, n=3).

\begin{tabular}{llllll}
\hline Sample & Element & Determined & Added & Found & Recovery $(\%)$ \\
\hline Drinking water & $\mathrm{Ru}$ & $\mathrm{ND}$ & 2.0 & $1.82 \pm 0.04$ & $91 \pm 2$ \\
& $\mathrm{Os}$ & $\mathrm{ND}$ & 2.0 & $2.05 \pm 0.07$ & $102 \pm 4$ \\
Tap water & $\mathrm{Ru}$ & $\mathrm{ND}$ & 2.0 & $1.96 \pm 0.06$ & $98 \pm 3$ \\
& $\mathrm{Os}$ & $\mathrm{ND}$ & 2.0 & $1.98 \pm 0.04$ & $99 \pm 2$ \\
\multirow{2}{*}{ River water } & $\mathrm{Ru}$ & $\mathrm{ND}$ & 2.0 & $1.80 \pm 0.03$ & $90 \pm 1$ \\
& $\mathrm{Os}$ & $\mathrm{ND}$ & 2.0 & $1.97 \pm 0.06$ & $99 \pm 3$ \\
Seawater & $\mathrm{Ru}$ & $\mathrm{ND}$ & 2.0 & $1.81 \pm 0.09$ & $90 \pm 4$ \\
& $\mathrm{Os}$ & $\mathrm{ND}$ & 2.0 & $1.97 \pm 0.10$ & $98 \pm 5$ \\
\hline
\end{tabular}

ND, not detected. 
Table S4. Comparison of the figures of merit for Os detection by PVGICPMS/ICPOES.

\begin{tabular}{lllll}
\hline Analyte & Medium & $\begin{array}{l}\text { Linearity } \\
\left(\mu \mathrm{g} \mathrm{L}^{-1}\right)\end{array}$ & $\begin{array}{l}\text { LOD } \\
\left(\mathrm{ng} \mathrm{L}^{-1}\right)\end{array}$ & Ref \\
\hline Os & $1 \% \mathrm{HAc}^{2} 50 \mathrm{ppm} \mathrm{Fe}^{2+/ 3+}$ & $0.2-3$ & 0.16 & {$[1]$} \\
Os & $5 \% \mathrm{HNO}_{3}$ & $0.1-10$ & 0.8 & {$[2]$} \\
Os & $\mathrm{H}_{2} \mathrm{O}$ & ${ }^{\mathrm{a}} 500-10000$ & ${ }^{\mathrm{a}} 200$ & {$[3]$} \\
$\mathrm{Ru}, \mathrm{Os}$ & $30 \% \mathrm{FA}^{2} 50 \mathrm{ppm} \mathrm{Co}^{2+}-20 \mathrm{ppm} \mathrm{Cd}^{2+}$ & $0.1-10$ & $5.0(\mathrm{Ru})$ & This work \\
\hline
\end{tabular}

a detected by ICP-OES

\section{References}

1. de Oliveira, R. M.; Borges, D. L. G.; Grinberg, P.; Sturgeon, R. E., Highefficiency photoreductive vapor generation of osmium. Journal of Analytical Atomic Spectrometry 2021, 36 (10), 2097-2106.

2. Gao, Y.; Li, S. Z.; He, H. Y.; Li, T. L.; Yu, T.; Liu, R.; Ni, S. J.; Shi, Z. M., Sensitive determination of osmium in natural waters by inductively coupled plasma mass spectrometry after photochemical vapor generation. Microchemical Journal 2017, $130,281-286$.

3. Zhu, Z.; He, D.; Huang, C.; Zheng, H.; Zhang, S.; Hu, S., High-efficiency photooxidation vapor generation of osmium for determination by inductively coupled plasma-optical emission spectrometry. Journal of Analytical Atomic Spectrometry 2014, $29(3), 506-511$. 